\title{
HEGEL, CONTRADICCIÓN Y DIALETHEIA ${ }^{1}$
}

\author{
Rafael Miranda Rojas \\ Pontificia Universidad Católica de Valparaíso \\ Universidad Bernardo O'Higgins \\ Universidad Santo Tomás \\ rafaelmirandarojas@gmail.com
}

\begin{abstract}
Resumen / Abstract
El presente escrito discute cómo Hegel comprende el principio de no contradicción. Se analiza, en primer lugar, el modo cómo Aristóteles entiende dicho principio, destacando las denominadas lecturas semántica y ontológica. Luego, se analiza si es correcto sostener que Hegel rechaza este principio, sosteniéndose aquí que, estrictamente, esto no es así. Para ello, se presenta una lectura dialetheista de la lógica hegeliana, la que permite enunciar que: i) Hegel no acepta el trivialismo; y, a partir de i), ii) no es viable un rechazo cabal del principio de no contradicción en la lógica hegeliana. El denominado contextualismo cognitivo permite descartar ciertas contradicciones como meras parameterizaciones, pero no conduce a la tesis fuerte de que Hegel rechaza el principio de no contradicción.

Palabras Clave: contradicción, Dialetheia, contextualismo cognitivo, parameterización.
\end{abstract}

\section{HEGEL, CONTRADICTION AND DIALETHEIA}

This paper discusses how Hegel understands the principle of contradiction. It is analysed, first, the way Aristotle understands this principle, emphasizing the so-called semantic and ontological readings. Then, it is analysed whether it is correct to argue that Hegel rejects this principle, holding here that, strictly speaking, this is not correct. To do this, we present a dialetheist reading of Hegelian logic, which allows to state that: i) Hegel does not accept trivialism and, from i), ii) is not feasible a full rejection of the principle of contradiction in the Hegelian logic. The so-called cognitive contextualism can dismiss some contradictions as mere parameterisations, but does not lead to the strong thesis that Hegel rejects the principle of contradiction.

KEY WORDS: contradiction, Dialetheia, cognitive contextualism, parameterisation.

1 La redacción de este escrito ha sido posible gracias al apoyo de la Comisión Nacional de Investigación Científica y Tecnológica (CONICYT), Becas Chile, Becas Pontificia Universidad Católica de Valparaíso y la Dirección de Investigación de la Universidad Bernardo O'Higgins. Una versión del mismo será presentada en el Congreso Internacional Hegel: A 200 años de la Ciencia de la Lógica, a desarrollarse entre el 12 y 14 septiembre del presente año en la Pontificia Universidad Católica de Valparaíso. Valparaíso, Chile. 


\section{Introducción}

$\overline{R M}$

La pregunta que guía este escrito es: ¿Rechaza Hegel el principio de no contradicción? El principio de no contradicción formalmente enuncia que $\neg$ $(\mathrm{p} \wedge \neg \mathrm{p})$, que no es el caso que $p$ y no-p. Brandom (2002) y McTaggart (1992), por ejemplo, sostienen que Hegel no rechaza este principio. Priest (2000), por otra parte, afirma que Hegel sí rechaza éste principio, siendo esto correcto desde las denominadas lógicas paraconsistentes, en las que es posible que sea verdadero $(\mathrm{p} \wedge \neg \mathrm{p})$. Ello no deriva en que, en todos los casos, $(\mathrm{p} \wedge \neg \mathrm{p})$ sea verdadero: ello sería afirmar que toda contradicción es verdadera. Admitir a lo menos una contradicción verdadera se denomina dialetheismo, aquella postura que sostiene que hay dialetheias: oraciones cuya afirmación y negación son verdaderas. Considerados diversos mundos posibles distintos del actual, el dialetheismo hegeliano requiere que al menos una contradicción sea verdadera en el mundo actual. El dialetheismo débil, por contraste, exige que al menos en un mundo posible una contradicción sea verdadera. Hegel (1991, p. 150) precisa en qué sentido la contradicción permite vitalidad dialéctica: "nothing else but the inner negativity of the determinations as their self-moving soul, the principle of all natural and spiritual life". Así entendida, la contradicción permitiría comprender las determinaciones en tanto negatividades, determinaciones que permiten proponer lo que Redding (2007, p. 210) denomina ley de no composibilidad de los contrarios (law of the non compossibility of contraries), es decir: la imposibilidad de que propiedades contrarias sean composibles. Más importante aún, permitiría no comprender el principio de no contradicción como lo que Redding (2007, p. 203) denomina el mito de lo dado lógico (myth of the logical given): que el principio de no contradicción puede ser conocido directamente a través de una intuición racionalista. Este escrito discute cómo la comprensión hegeliana difiere de la aristótelica expresada en Peri Hermeneias, enfatizando los aspectos en común de ambas posturas. Particularmente, analiza cómo, si hay un rechazo al principio de contradicción, lo es en vistas a lo que Redding (2007, p. 204) denomina contextualismo cognitivo (cognitive contextualism), es decir: que Hegel acepta un principio de contradicción contextualmente determinado, acorde con la comprensión dialéctica del mismo. Finalmente, se argumenta cuál sería la principal consecuencia metafísica de esta postura: el anti realismo.

El desarrollo de este escrito es el siguiente: La sesión 2 presenta la comprensión aristotélica y hegeliana del principio de no contradicción, discutiendo particularmente dos aristas: la ontológica y la semántica. La sesión 3 discute de qué modo la lectura hegeliana del principio de no contradicción puede ser comprendida desde una postura dialetheista. La sesión 4 analiza los alcances metafísicos de la propuesta hegeliana ante el principio de no contradicción, evidenciando una cercanía con la lectura aristotélica a partir del principio de no composibilidad de los contrarios. A su vez, enuncia su diferencia central: el anti realismo. La sesión 5 analiza la alternativa contextualista, explicitando como esta puede ser comprendida como un caso de parameterización. Finalmente, en la sesión 6 se explicitan las principales conclusiones. 


\section{El principio de contradicción: ontología y semántica ${ }^{2}$}

\subsection{Aristóteles}

El principio de no contradicción es enunciado claramente en dos textos del corpus aristotélico: Metafísica y Peri Hermeneias ${ }^{3}$. Si bien puede de entrada sostenerse que en ambos textos es enunciado el mismo principio, en Metafísica lo es desde una arista ontológica, mientras que en el Peri Hermeneias lo es desde una arista semántica ${ }^{4}$. En Metafísica IV $(\Gamma)$, Aristóteles afirma:

"It is imposible for the same thing to belong and not to belong at the same time to the same thing and in the same respect" $t^{5}$ (Metafísica IV 3 1005b 19-20).

Pasaje en el que se aprecia cómo Aristóteles sostiene el principio de no contradicción en vistas a lo qué es algo ( $\tau$ ivós), y qué propiedades le corresponden a ese algo. Por su parte, en Peri Hermeneias 17 a 30 se enuncia dicho principio del siguiente modo: кaì

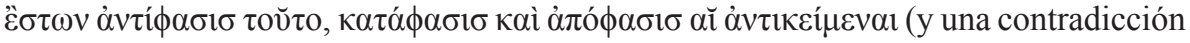
es esto: la afirmación y la negación opuestas). Se observa que en este pasaje se sostiene, dicho de una oración ( $\lambda$ ơós), que el principio de contradicción corresponde a la afirmación y negación de la misma. Es importante observar cómo este segundo modo de comprender el principio depende o descansa en el primero, pues acepta que

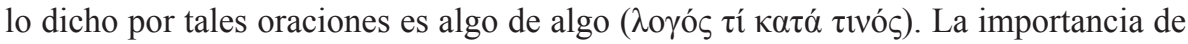

2 Junto con la lectura ontológica y semántica del principio de no contradicción, cabe mencionar la denominada lectura psicológica. Es explicitada en Metafisica IV 1005b 23-25 y dice lo siguiente: "It is imposible for anyone to believe that the same thing is and is not, as some consider Heraclitus said" (énfasis de quien escribe) Este modo de comprender el principio de no contradicción puede denominarse también doxástico, por la alusión explícita al estado mental de cierto sujeto $S$, la creencia. Debe mencionarse, sobre este modo de entender el principio, que Aristóteles no distingue entre que $S$ crea $p$, y que $S$ crea $p$ justificadamente, o con razón. Todo parece indicar que, en el primer caso (que $S$ crea $p$ simpliciter), sí es admisible que $S$ tenga creencias contradictorias, pero no creencias contradictorias justificadas.

3 También se encuentra en Analíticos Posteriores, pero tal texto no será considerado en lo que sigue.

4 En Metafísica, luego del enunciado citado líneas abajo, Aristóteles también presenta una lectura semántica del principio de no contradicción, a saber: "Opposite assertions cannot be true at the same time" (Metafísica IV 6 1011b 13-20). No obstante, el pasaje en el que enuncia del modo más claro la lectura semántica del discutido principio, es en Peri Hermenias.

5 Así enunciado, el principio de no contradicción es explícitamente modal: enuncia lo que es imposible de una misma cosa. Es importante explicitar esto, pues evidencia el nexo cercano en la lógica aristotélica entre el principio de no contradicción y el principio de no composibilidad de los contrarios, que será discutido ulteriormente. Una formalización que muestra este carácter modal se encuentra en Wedin (2004a), a saber: $\neg \diamond(\exists x)(F x \wedge \neg F x)$. 
este principio, en el ámbito epistémico, reside en que al parecer sin éste no podría conocerse. Aristóteles lo enuncia del siguiente modo: "is necessary for anyone to have who knows any of the things that are" (Metafisica IV 3 1005b 15).

Las consecuencias epistémicas del rechazo de este principio serían desastrosas: no podría conocerse qué es algo, ni qué propiedades le corresponden a ese algo. Por otra parte, es un punto en discusión qué principio es el primordial en la concepción aristotélica: el de no contradicción o el de identidad ${ }^{6}$. Esto, pues al menos en el modo cómo comprende el principio en Metafísica, el principio de contradicción asume que una cosa es idéntica consigo misma y, además, no tiene propiedades opuestas en un tiempo $t^{7}$. No obstante esto, Aristóteles acepta que el principio de no contradicción no tiene demostración. Si la tuviese, ello derivaría en un regreso al infinito, tal que todo principio debiese estar del mismo modo sujeto a demostración.

Un aspecto central del modo cómo Aristóteles entiende el principio de no contradicción en su arista ontológica es el denominado esencialismo: que cierta(s) propiedad(es) le(s) corresponda(n) necesariamente a un objeto $o$. Una estrategia que evidencia este compromiso esencialista en Aristóteles es la aplicación del método eléntico ${ }^{8}$ o de reducción al absurdo (reductio ad absurdum) como una vía de justificación

$6 \quad$ Puede incluirse en este debate el principio de tercero excluso. Sobre este principio, Aristóteles enuncia que: "Of any one subject, one thing must be either asserted or denied" (Metafisica 1011b 24). Pueden distinguirse ambos principios, de no contradicción y de tercero excluso, desde los distintos tipos de oposición propuestos por Aristóteles. Particularmente, contradicción y contrariedad. Cabe considerar que en ambas oposiciones, Aristóteles intenta evidenciar la incompatibilidad de verdad y falsedad. La diferencia es que en la relación de oposición denominada contradicción, la incompatibilidad es exhaustiva: Si p es verdadero, no-p es falso. Un ejemplo es "Ella está sentada" y "Ella no está sentada", casos en los que si una oración es verdadera, la otra es falsa. Por lo tanto, en esta relación de oposición se aplica el principio de tercero excluso. En contraste, la oposición denominada contrariedad, cuyo par de enunciados son denominados contrarios (a diferencia de los contradictorios), si bien se oponen, no es una oposición exhaustiva. Ello quiere decir que en la contrariedad se cumple el principio de no contradicción, pero no el de tercero excluso: puede darse el caso de que ambas oraciones sean falsas, si bien no se puede dar el caso que ambas sean verdaderas. Un ejemplo es "Pedro está feliz" y "Pedro está triste". Sobre este último punto sostiene Aristóteles: "Nothing can exist between two contradictories, but something may between contraries" (Metafísica 1055b 2). El principio de tercero excluso puede ser formalizado del siguiente modo: $(\mathrm{p} \vee \neg \mathrm{p})$.

7 Sobre la relación estrecha entre el principio de contradicción y el principio de identidad, cabe mencionar la denominada ley de indiscernibilidad de los idénticos, enunciada por Leibniz (1696). Si x e y son idénticos, entonces tienen las mismas propiedades. Por contraste, si x e y no son idénticos, entonces son discernibles: no comparten al menos una propiedad.

8 Este método es considerado estrictamente socrático. Para lo que interesa en este escrito, es analogable con el denominado argumento trascendental propuesto por Kant (1781). El nexo entre ambos es que se asume como correcto el principio de no contradicción o, para el caso del argumento trascendental, que si ciertos aspectos de la experiencia son posibles, el mundo debe ser de cierta manera; dado que dichos aspectos existen, el mundo debiese ser 
de que el principio de no contradicción es correcto: no puede afirmarse de un objeto $o$, que es $F$ y que no es $F$ al mismo tiempo y bajo el mismo respecto.

Semánticamente, el principio de no contradicción es formalizado actualmente del siguiente modo: $\neg(\mathrm{p} \wedge \neg \mathrm{p})$. Ello no es, estrictamente, lo que Aristóteles intentaba enunciar. La diferencia es que, en esta formalización, $p$ es comprendida como una proposición completa, la cual es negada o afirmada según sea el caso. Por contraste, lo que enuncia Aristóteles es cómo un cierto predicado le corresponde o no a cierto objeto: este matiz corresponde a la diferencia entre la lógica proposicional y la lógica comprendida desde los términos que se encuentran en cierta proposición ${ }^{9}$. No obstante esto, la idea central persiste: no es viable sostener $p$ y no- $p$, como no lo es sostener que una propiedad $F$ y su negación, no- $F$, le corresponden a un mismo objeto $o$ en un mismo tiempo $t$, bajo el mismo respecto. Es importante destacar, además, que para Aristóteles la contradicción se da solo en la relación entre enunciados, no entre términos. En Categorías 13b3-12 el filósofo griego se ocupa de este punto, al establecer una condición previa para que un par de predicados, $F$ y no- $F$, sean contradictorios, a saber: que de aquello que son predicados, exista. Sostiene Aristóteles, dicho del par de enunciados "Sócrates está enfermo" y "Sócrates está sano" lo siguiente:

"If Socrates exists, one will be true and the other false, but if he does not exist, both will be false; for neither 'Socrates is sick' nor 'Socrates is well' will be true, if Socrates does not exist at all” (Categorías 13b26-35).

\subsection{Hegel}

Como lo enuncia Houlgate (2009, p. 112), la Ciencia de la Lógica y la Enciclopedia Lógica tratan sobre las denominadas categorías o formas del pensamiento (Denkformen). Hegel dice de las categorías, en la Enciclopedia Lógica, que son:

“...what we are most familiar with (das Bekannteste): being, nothing, etc.; determinacy, magnitude, etc.; ...one, many, and so on". Hegel (1991, 45, $\$ 19)$.

de tal manera. La idea central es que el mundo determina las experiencias que son posibles, siendo un caso paradigmático a favor del principio de no contradicción el que un objeto o no pueda ser $\mathrm{F}$ y no - F en un mismo tiempo t. De todos modos, este método no está exento de crítica, pues en el caso de lo que es pensado por un sujeto $\mathrm{S}$, cabe plantearse la pregunta de si el mundo es de cierto modo porque tal sujeto lo piense de tal modo, o si por contraste solo es viable sostener que tal sujeto $\mathrm{S}$ piensa que el mundo es de cierto modo, sin que ello exija que deba ser así.

$9 \quad$ Esto es observado por Redding (2007, p. 204) al afirmar: "it is an anachronism to regard Aristotle as here referring to a pair of contradictory propositions ( $p$ and $\ulcorner p$ ) as understood in modern logic". 
Considerada la distinción ontológica-semántica enunciada anteriormente desde la postura aristotélica, cabe preguntarse cuál es el modo correcto de entender la postura que Hegel tiene ante el principio de no contradicción: ¿rechaza la ontología aristotélica; rechaza el modo cómo se comprende una oración, su aspecto semántico; o ambas? Esta cuestión es central para entender correctamente lo enunciado por Hegel en la Ciencia de la Lógica, como en otros escritos del corpus hegeliano. Dice el filósofo alemán:

"Contradiction is the root of all movement and vitality; it is only in so far as something has a contradiction within it that it moves, has an urge and activity" (Hegel 1969, p. 439).

Esta vitalidad que enfatiza Hegel establece la distinción entre Verstand y Vernunft, entendimiento y razón, lo que parece ser una crítica epistémica al principio de no contradicción. Una razón por la que es importante establecer qué es lo que afirma Hegel al sostener esto es si intenta defender que toda contradicción es semánticamente verdadera o si, por contraste, intenta establecer el modo cómo la relación de contradicción permite determinar lo que un objeto es, y lo que no-es. Esta falta de vitalidad la explica Hegel en un pasaje previo del mismo escrito:

“...its determinations are accepted in their unmoved fixity and are brought only into an external relation with each other. In judgements and syllogisms the operations are in the main reduced to and founded on the quantitative aspect of the determinations; consequently everything rests on an external difference, on mere comparison and becomes a completely analytical procedure and mechanical (begriffloses) calculation" (ídem., p. 52).

Así comprendido el análisis lógico en general, y el principio de no contradicción en particular, constituyen casos de una determinación fija, no dialéctica. Según Redding (2007, p. 200), el principio que guía la comprensión hegeliana de la contradicción es que "todo es inherentemente contradictorio". ¿Cómo debe entenderse esta afirmación? Hegel comprende este principio como aquel que establece la verdadera y esencial naturaleza de las cosas (ídem., p. 439). Es, evidentemente, la defensa de una postura ontológica. No intenta en primer lugar sostener una particular tesis frente al caso de dos oraciones opuestas. No obstante, en el plano ontológico es claramente una concepción distinta de la aristotélica. Ante la tesis de Aristóteles de que nada es contradictorio se observa la tesis de que todo es contradictorio.

Aun cuando el rechazo del principio de no contradicción parece incuestionable, ciertos autores contemporáneos descartan que Hegel acepte tal lectura. Un motivo para evitar atribuir esta posición a Hegel, es una consecuencia implausible del rechazo del principio de no contradicción: la denominada consecuencia explosiva, que tal rechazo implique irracionalismo, tal que de una contradicción se siga cualquier proposición. Brandom (2002, p. 178) en vistas a explicar por qué en un sentido fuerte Hegel no rechaza el principio de no contradicción, aborda qué quiere decir que el mundo esté determinado. Según Brandom, un objetivo central de Hegel es comprender cómo son las cosas. Para ello, las cosas deben estar determinadas: "there must be a distinction 
between things being that way and their being some other way". Es una característica, sostiene Brandom, objetiva de las cosas. El que sean definidas o determinadas quiere decir que algo las diferencia. Estas determinaciones permiten por tanto individualizar e identificar tales cosas, tales objetos. Que los objetos estén determinados quiere decir que tienen diferencias entre ellos. Para que un objeto $x$ sea distinto de un objeto $y$, ciertas determinaciones que tiene $x$ no deberían corresponderle a $y$, o viceversa. Brandom distingue entre meras (mere) diferencias y diferencias exclusivas. Meras diferencias apunta a determinaciones compatibles entre sí, como ser "verde" y "cuadrado"; mientras que diferencias exclusivas son aquellas que no son compatibles entre sí, como "cuadrado" y "triangular". Lo importante es que, apunta Brandom, esta distinción es sostenida por Hegel y, más importante aún, rechaza que el mundo, cómo son las cosas, esté determinado solo por meras diferencias:

"Hegel argues that the idea of a world exhibiting definiteness or determinateness as mere (gleichgultige) difference, without exclusive (auschliessende) difference, is incoherent. This is why compatibly different properties always come as members of families of exclusively different ones" (ibíd.).

Brandom observa en las diferencias exclusivas un modo de negación acorde con el principio de no contradicción. Si $p$ es una determinación que excluye no- $p$, sería correcto establecer que $p$ excluye no- $p$.

Pero ante esta posición, cabe mencionar la de Priest (2002), para quien no es correcto sostener que Hegel acepta el principio de no contradicción, sino que estrictamente lo niega. Negar el principio de no contradicción es característico de las denominadas lógicas paraconsistentes: sistemas lógicos que permiten que al menos en un caso, $p$ y $n o-p$ sean ambos verdaderos. Estos sistemas lógicos permiten resolver paradojas como la denominada paradoja del mentiroso (liar paradox), que sea verdadero el enunciado "Esta oración es falsa". No obstante, Redding (2007, p. 207) precisa que no basta proponer la lectura hegeliana como un caso de lógica paraconsistente. No basta, pues tales sistemas no sostienen como un tesis subsidiaria que el mundo actual $M$ tiene al menos un caso de contradicción verdadera, sino que en al menos un mundo posible, $M 2$, ocurre esto. Hegel, por contraste, se ubicaría en la postura denominada dialetheismo fuerte: que en este mundo actual $M$ hay contradicciones.

\section{Dialetheia}

“...it is the requirement and the business of logical thinking to enquire into just this, whether such a finite without infinity is something true, or whether such an abstract infinity, also a content without form and a form without content, an inner by itself which has no outer expression, an externality without an inwardness, whether any of these is something true or something actual" (Hegel 1969, pp. 41-42). 
El dialetheismo tiene como tesis central que hay un par de proposiciones, $p$ y no- $p$, tal que ambas son verdaderas ${ }^{10}$. Comprender la lógica hegeliana como un caso de esta postura exigiría, por tanto, que de algún modo su análisis de las categorías considere lo que es enunciado, lo que es dicho, como una manifestación de lo que es y lo que no es en sus determinaciones, las categorías. Ello no sería errado, si se considera lo observado por Houlgate (2009, p. 112): que las categorías del pensamiento (Denkformen), si bien no deben confundirse con las palabras a través de las que son expresadas, es por medio de estas que se accede epistémicamente a las primeras. Lo enuncia Hegel al sostener "we think in names" (Hegel 1971, 220 \$462). El caso paradigmático de oposición de proposiciones es la contradicción. Si el dialetheismo acepta casos en que la oposición de $p$ y no- $p$ es verdadera, acepta casos en que el principio de no contradicción no se cumple ${ }^{11}$ : casos en que ( $\mathrm{p} \wedge \neg \mathrm{p}$ ) es verdadero: las denominadas dialetheias. El dialetheismo permite que la relación de implicación, la inferencia deductivamente válida, sea comprendida como paraconsistente, es decir: que acepte como válido que de $(\mathrm{p} \wedge \neg$ p) se siga $q$. No obstante, esto no es estrictamente lo mismo que aceptar contradicciones verdaderas simpliciter. Un objetivo de la lógica paraconsistente es evitar la consecuencia denominada explosiva, tal que toda contradicción sea verdadera y, específicamente, que de una contradicción se siga cualquier proposición (ex contradictione quolibet). Un aspecto que debiese enfatizarse es cómo el dialetheismo y la lógica paraconsistente, comprendidas de este modo, no son un rechazo cabal del principio de no contradicción. Es esto lo que las diferencia del denominado trivialismo (ver nota anterior). De este modo, el dialetheismo no se vería forzado a aceptar que:

i) toda contradicción es verdadera y

ii) que de una contradicción se sigue cualquier proposición: $(\mathrm{p} \wedge \neg \mathrm{p}) \rightarrow \mathrm{q}$.

Siguiendo a Priest (2008), es importante destacar la principal diferencia entre lógica paraconsistente y dialetheismo. Si bien es cierto ambas posturas intentan evitar el trivialismo, la consecuencia explosiva atribuida al rechazo del principio de no contradicción, la lógica paraconsistente no requiere para lograr esto aceptar que hay contradicciones verdaderas simpliciter, o verdaderas en el mundo actual: puede aceptar que son posibilidades reales (la postura denominada strong paraconsistency) en algún mundo posible, tal que en dicha situación contrafáctica sea verdadera cierta contradicción; o puede rechazar que una contradicción sea una posibilidad real, y admitir solamente su utilidad en ciertos ámbitos científicos, como las matemáticas (week paraconsistency). El dialetheismo, por contraste, acepta la tesis fuerte de que en el mundo actual hay al menos un caso de contradicción verdadera.

10 Como precisa Priest (2008), un segundo modo de comprender la postura dialetheista es que $\mathrm{p}$ sea verdadero y falso. Este modo de comprender el dialetheismo tiene como tesis subsidiaria que falsedad se entiende como la verdad de la negación.

11 Si la tesis es que toda contradicción es verdadera, se denomina trivialismo. Ver Priest (2008). 
No es extraño proponer que el modo cómo Hegel comprende el principio de no contradicción es dialetheista. Los antecedentes del dialetheismo permiten esta afirmación, considerando que uno de los fragmentos más famosos de Heráclito es interpretado como un caso de dialetheia. Tal fragmento es el 49a: "We step and do not step in the same rivers; we are and we are not” (Robinson 1987, p. 35) ${ }^{12}$. En la Ciencia de la Lógica, Hegel explícitamente sostiene una postura dialetheista al afirmar:

"Something moves, not because at one moment it is here and another there, but because at one and the same moment it is here and not here, because in this 'here', it at once is and is not" (Hegel 1971, p. 440).

El antecedente directo por el que Hegel considera correcto sostener que la realidad puede ser comprendida como inconsistente es Kant (1781). Específicamente, a partir del modo cómo Kant comprende las denominadas antinomias de la razón. Sucintamente, Kant comprendía las antinomias de la razón como un intento por superar los límites del conocimiento. Conceptos como antes de, parte de, causa de, son comprendidos como una medida objetiva de lo que puede conocerse, cometiendo el error, según la perspectiva kantiana, de confundir una condición epistémica subjetiva con una característica objetiva del mundo (Kant 1781, 300). Hegel acepta que la contradicción se entienda como determinación del pensamiento, entendiendo las antinomias como lo que él denomina la objetividad de la ilusión y la necesidad de la contradicción (Hegel 1971, p. 56). Lo que Kant comprende como una determinación del pensamiento, Hegel lo entiende como una característica no solo de la razón, sino del mundo actual: la naturaleza dialéctica del mundo. Según Redding (2007, p. 202), es esto lo que diferencia la postura kantiana de la hegeliana:

"Kant, of course, had not embraced the contradictions generated by the operations of reason, and did not draw Hegel's seemingly irrationalist metaphysical conclusion that of the contradictoriness of all things. Rather, he saw it as marking the limits within which reason had to self-consciously restrain itself..."

Como se observa, Redding atribuye a Hegel un compromiso con la consecuencia explosiva, el irracionalismo que exigiría aceptar toda contradicción en el mundo actual. Por otra parte, evidencia la postura de no aceptar que el principio de no contradicción sea comprendido como lo que se denomina logical given: la posición que acepta un acceso epistémico intuicionista o, en otras palabras, no inferencialista con respecto al principio de no contradicción.

12 Aristóteles, al proponer la lectura doxástica del principio de no contradicción, ataca otro caso paradigmático de dialetheia, el relativismo defendido por Protágoras: que el hombre es la medida de todas las cosas. 


\section{Contradicción, no composibilidad de los contrarios y anti realismo}

Un aspecto de la lógica aristotélica, que es analizado por Redding (2007), es el nexo estrecho entre el principio de no contradicción y el principio de no composibilidad de los contrarios. Intuitivamente podría afirmarse que se acerca a lo revisado a partir de Brandom (2002), como una defensa del principio de no contradicción en la lógica hegeliana. Tomando un ejemplo de Redding (2007, p. 213), parece correcto sostener que si un objeto $o$ es determinado como rojo, o como siendo de color rojo, podría afirmarse además, entre otras de sus determinaciones, no ser verde. Si un sujeto $S$ afirma "Esta manzana es roja", podría correctamente sostener "Esta manzana no es verde". ¿Son ambas determinaciones de la manzana en este caso? La respuesta a esta pregunta supondrá, entre otros aspectos, una postura ante: a) si la manzana es una sustancia de la cual se predican ciertas propiedades y b) si determinaciones como no - verde son propiedades de, en este caso, la manzana. El punto es importante, pues evidencia cómo son comprendidas determinaciones como no ser verde. Aristóteles enuncia una crítica cercana a este punto contra Heráclito, al decir: "...it is impossible for contrary attributes to belong at the same time to the same subject" (Metafisica IV,1005b 27-33). Rojo y no-verde, como también rojo y no-rojo, y rojo y verde, son casos de determinaciones opuestas desde la lectura aristotélica. Si bien Redding no acepta que la lógica hegeliana sea sustancialista en este punto, siguiendo a Brandom es discutible que Hegel aceptara que una manzana, este objeto específico que existe y del que un sujeto $S$ afirma "Esta manzana es roja" y "Esta manzana no es verde", afirmase a su vez como una caraterística de este objeto $o$, ser rojo y no-rojo. La dialéctica hegeliana, según esta lectura, no tendría por objetivo rechazar la no composibilidad de rojo y no-rojo (un caso de contrarios), sino la válida determinación negativa de no-verde dicho de la misma manzana. Como se ha mencionado, para Brandom determinación supone identidad e individuación. La diferencia está en que se comprende que Hegel no tiene por objetivo considerar los objetos separados de sus relaciones, sino más bien comprender el mundo desde estas relaciones. Así, la diferencia es que Hegel no considera correcto determinar esta manzana, este objeto, separado de sus relaciones con el mundo: el denominado holismo. Luego: ¿es correcto desde esta postura sostener que tanto rojo como no-rojo sean determinaciones de esta manzana? Como explicita Brandom, Hegel comparte la posición de Spinoza de que toda determinación es negación (omnis determinatio est negatio). Por ello Brandom piensa que Hegel no rechaza el principio de no contradicción, pues es el que evidencia claramente esta característica de las determinaciones:

"For an essential, defining property of negation is the exclusiveness codified in the principle of non contradiction: $p$ rules out not-p; they are incompatible. For Hegel, it is this exclusiveness that is the essence of negation. He abstracts this feature from the case of formal negation, and generalizes it to include the sort of material incompatibility that obtains between the properties square and triangular. (Formal negation can then reappear as the shadow of material incompatibility: not-p is the minimal incompatible of $\mathrm{p}$. It is what is entailed by everything materially incompatible with p.) In a conceptually deep sense, far from rejecting the law of non contradiction, I want to claim that Hegel radicalizes it, and places it at the very center of his thought" (Brandom 2002, p. 179). 
No es complejo, a partir de esto, observar que rojo y no rojo son excluyentes entre sí, mientras que rojo y no-verde no: un objeto puede cumplir con el segundo par de determinaciones a la vez, pero no con el primero. Sin embargo, todo parece indicar que no puede comprenderse a Hegel como dialetheista y a la vez como aceptando el principio de no composibilidad de los contrarios. Esto sería así, pues tal como observa Priest (2008), el dialetheismo parece estar comprometido con la existencia de al menos un objeto inconsistente: que tenga entre sus características tanto $p$ como no- $p^{13}$.

Dado que lo que intenta Hegel no es solamente establecer las categorías lógicas de modo abstracto sino sostener que es una característica del mundo el que todo sea inherentemente contradictorio, es viable sostener que la lógica hegeliana, en tanto parte del programa idealista del filósofo alemán, es un caso de anti realismo. Por realismo se entiende aquella posición metafísica que afirma la existencia de entes, independiente de los estados mentales de un sujeto $S$. Específicamente, esta posición sostiene que las características de un objeto no dependen de que un sujeto $S$ las enuncie ni las piense. El modo en que la comprensión dialetheista sería un caso de anti realismo radicaría en que una categoría del pensamiento, una determinación lógica como la admisión de contradicciones verdaderas, sería una característica del mundo ${ }^{14}$. Una vía que permite evitar esto sería sostener que no son aplicables al mundo categorías mentales como consistencia e inconsistencia, de tal modo que no fuese estrictamente correcto afirmar la existencia ya de objetos consistentes, ya de objetos inconsistentes. Priest (2008) sugiere esto. No obstante, también es viable sostener un dialetheismo semántico, tal que se acepten contradicciones verdaderas, sin que ello exija sostener la existencia de objetos inconsistentes; o también aceptar hechos, estados de cosas, o eventos negativos, de modo tal que en el mundo actual haya truth makers (hacedores de verdad) tanto para $p$ como para no- $p$. De este modo, sería el caso que $p$ y que no- $p$, comprendidos ambos como estados de cosas, no como proposiciones.

\section{Contextualismo cognitivo y dialéctica}

"Contradiction for Hegel is a necessary concomitant to his cognitive contextualism, and to the necessarily 'heterogeneous' conception of logic that this contextualism brings with it. For Hegel, we might say, there is no one 'law of non-contradiction' that could be affirmed or rejected as normative for all thought. Given the fact that Hegel calls upon the underlying structures of both term and propositional

13 Sin los casos usuales de parameterización, como el que apela a diferencias temporales. Ver apartado siguiente.

14 Ver Beiser (2008, p. 6), quien afirma sobre este punto: "the identity of subject and object, not only how we think about the world but the world itself". De todos modos, debe observarse como, así comprendido el anti realismo, permitiría enunciar la misma crítica a la postura aristotélica: que se comprenda el principio de contradicción como determinando lo que el mundo es. 
logic, understanding what counts as contradiction for him will presuppose an understanding of how the concept of contradiction changes between its ancient and modern expressions" (Redding 2007, p. 204).

Si el dialetheismo no exige el rechazo cabal del principio de no contradicción, cabe preguntarse de qué modo Hegel puede admitirlo. Redding sostiene que el contextualismo epistémico explicaría cómo, desde las circunstancias de dos sujetos $S 1$ y $S 2$, sería incorrecto exigir que ambos afirmasen, v.gr., "Londres es bella", si no es el caso que ambos creen esto.

Este contextualismo tiene, no obstante, un nexo cercano con la denominada parameterización (parameterisation): defender la validez del principio de no contradicción, a partir de que los casos putativos en que no se cumplan correspondan a ambigüedades del lenguaje o a usos metafóricos del mismo. Si dos proposiciones $p$ y no- $p$ tuviesen significados distintos, no podrían por este motivo ser comprendidas como contradictorias. Si bien es cierto, en el caso del contextualismo epistémico no se discute que dos sujetos $S 1$ y $S 2$ den un significado diferente a la proposición "Londres es bella", si se exige que para que se afirme o se niegue esta proposición, los contextos de ambos sujetos sean distintos. De todos modos, el punto es que el mentado dialetheismo hegeliano, para que no derive en trivialismo, debe aceptar casos, estados de cosas o eventos, en que sí se cumpla el principio de no contradicción. Esto es importante, pues evidencia un modo de aceptar el principio de no contradicción, sin que se entienda esto como una apelación a lo dado lógico (logical given).

\section{Conclusión}

El análisis precedente ha permitido observar que no es correcto atribuir a Hegel un rechazo cabal del principio de no contradicción. Ello no es así, por las consecuencias contra intuitivas que se derivarían de tal rechazo, a saber: trivialismo e irracionalidad (la consecuencia explosiva). Conjuntamente, el nexo entre las categorías del pensamiento (Denkformen) y la determinación del mundo evidencia un compromiso anti realista de la lógica hegeliana.

\section{Referencias bibliográficas}

Ackrill, J. L. (1963), Aristotle’s Categories and De Interpretatione. Traducción y Comentarios. Oxford: Clarendon Press.

Aristóteles (1980), Peri Hermeneias. Traducción Alfonso García Suarez y Julian Velarde Lombraña. Teorema.

Barnes, J. (1975), Aristotle’s Posterior Analytics. Traducción y Comentarios. Oxford: Clarendon Press. 
Beiser, F. (2008), "The Puzzling Hegel Renaissance", The Cambridge Companion to Hegel and Nineteenthcentury Philosophy, pp. 1-14. Edit. Frederick C. Beiser. Cambridge University Press.

Brandom, R. (2002), Tales of the Mighty Dead: Historical Essays, The Metaphysics of Intentionality. Cambridge, Mass: Harvard University Press.

Hegel, G. W. F. (1969), Science of Logic. Traducción. A. V. Miller. London: Allen and Unwin.

(1971), Hegel's Philosophy of Mind. Trad. W. Wallace. Oxford Clarendon Press.

(1991), The Encyclopaedia Logic: Part I of the Encyclopaedia of Philosophical Sciences. Traducción. T. F. Geraets, W. A. Sutching, \& H. S. Harris. Indianapolis: Hackett.

Houlgate, S. (2009), "Hegel's Logics", The Cambridge Companion to Hegel and Nineteenthcentury Philosophy, pp. 111-134. Edit. Frederick C. Beiser. Cambridge University Press.

Jaeger, W. (1951), Aristotelis Metaphysica. Oxford Classical Text. Oxford: Clarendon Press.

Kant, I. (1781), Kritik der reinen Vernunft. Traducción inglesa: Critique of Pure Reason. New York: Palgrave Macmillan, 2003.

Leibniz, Gottfried (1696), New Essays Concerning Human Understanding by Gottfried Wilhelm Leibniz. A. G. Langley, ed. and trans., 2d ed., Chicago: Open Court, 1916.

McTaggart, J. (1992), Studies in the Hegelian Dialectic. Segunda edition.Cambridge University Press.

Priest, G. (2000), “Could Everything Be True?”. Australasian Journal of Philosophy. 78: $189-95$.

(2002), Beyond the Limits of Thought. Oxford University Press. (2006), Doubt Truth to Be a Liar. Oxford University Press. (2008), "Dialetheism", The Stanford Encyclopedia of Philosophy. http:// plato.stanford.edu/entries/dialetheism/.

Redding, P. (2007), Analytic Philosophy and The Return of Hegelian Thought. Cambridge University Press

Robinson, T. M. (1987), Heraclitus: Fragments. Toronto: University of Toronto Press.

Wedin, Michael V. (2004a), "Aristotle on the firmness of the principle of noncontradiction" Phronesis 49: 226-65.

(2004b), "On the use and abuse of non-contradiction: Aristotle's critique of Protagoras and Heraclitus in Metaphysics Gamma 5", Oxford Studies in Ancient Philosophy 26: 213-39. 